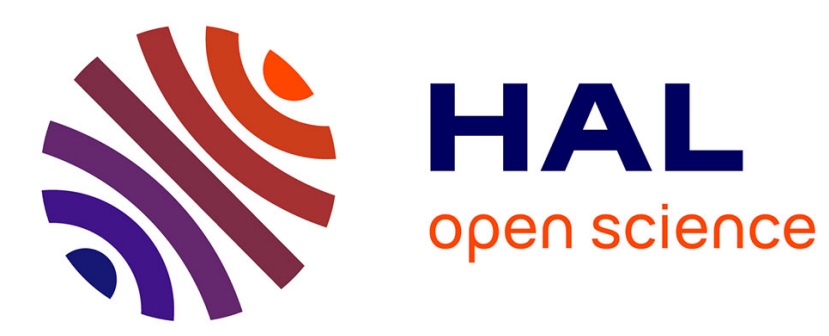

\title{
Indeterminacy and business-cycle fluctuations in a two-sector monetary economy with externalities
}

Stefano Bosi, Kazuo Nishimura, Alain Venditti

\section{To cite this version:}

Stefano Bosi, Kazuo Nishimura, Alain Venditti. Indeterminacy and business-cycle fluctuations in a two-sector monetary economy with externalities. 2009. halshs-00432268

\author{
HAL Id: halshs-00432268 \\ https://shs.hal.science/halshs-00432268 \\ Preprint submitted on 15 Nov 2009
}

HAL is a multi-disciplinary open access archive for the deposit and dissemination of scientific research documents, whether they are published or not. The documents may come from teaching and research institutions in France or abroad, or from public or private research centers.
L'archive ouverte pluridisciplinaire HAL, est destinée au dépôt et à la diffusion de documents scientifiques de niveau recherche, publiés ou non, émanant des établissements d'enseignement et de recherche français ou étrangers, des laboratoires publics ou privés. 


\title{
GREQAM
}

Document de Travail

Groupement de Recherche en Economie Quantitative d'Aix-Marseille - UMR-CNRS 6579 Ecole des Hautes Etudes en Sciences Sociales Universités d'Aix-Marseille II et III

INDETERMINACY AND BUSINESS-CYCLE FLUCTUATIONS IN A TWO-SECTOR ECONOMY WITH EXTERNALITIES

\author{
Stefano BOSI \\ Kazuo NISHIMURA \\ Alain VENDITTI
}

September 2009

$n^{\circ} 2009-26$ 


\title{
Indeterminacy and business-cycle fluctuations in a two-sector monetary economy with externalities ${ }^{1}$
}

\author{
Stefano BOSI \\ EQUIPPE, University of Lille 1 \\ Kazuo NISHIMURA \\ Institute of Economic Research, Kyoto University \\ and \\ Alain VENDITTI \\ $C N R S$ - GREQAM and EDHEC
}

\begin{abstract}
We consider a two-sector economy with money-in-the-utility-function and sector-specific externalities. We provide conditions on technologies leading to the existence of local indeterminacy for any value of the interest rate elasticity of money demand, provided the elasticity of intertemporal substitution in consumption is large enough. Moreover, we show that the occurrence of multiple equilibria is intimately linked with the existence of a flip bifurcation and period-two cycles.
\end{abstract}

Keywords: Money-in-the-utility-function, two-sector economy, sector-specific externalities, indeterminacy, period-two cycles, sunspot equilibria.

Journal of Economic Literature Classification Numbers: C61, E32.

\footnotetext{
${ }^{1}$ This paper is dedicated to 65 year-old birthday of our dear friend and great scientist, Saber Elaydi.
} 


\section{Introduction}

Endogenous business-cycle fluctuations through the existence of local indeterminacy are known to occur in monetary growth models in which the central bank is assumed to follow an exogenous money growth rule. Particular examples are provided by Fukuda [6], Matsuyama [7] and Woodford [12] within optimal growth models with money-in-the-utility-function (MIUF). Local indeterminacy means that there exists a continuum of equilibria starting from the same initial condition, all of which converging to the same attractor such as a steady state. It is now well-known that local indeterminacy is a sufficient condition for the existence of endogenous fluctuations generated by purely extrinsic shocks on beliefs which do not affect the fundamentals, i.e. preferences and technologies. ${ }^{2}$ Indeed, in presence of local indeterminacy, randomizing beliefs over a continuum of equilibrium paths generates new stochastic equilibria derived from shocks on expectations. These expectations-driven fluctuations are no longer based on exogenous shocks on fundamentals and represent a convincing rationale to explain macroeconomic volatility through alternative propagation mechanisms.

Formally, local indeterminacy arises when the stable manifold has dimension greater than the number of predetermined variables (i.e., determined the period before and currently taken as given). Within traditional MIUF models, the competitive equilibrium is described by a system of implicit difference equations of order 2 in the capital stock $k$, namely in $k_{t}, k_{t+1}$ and $k_{t+2}$, and order 1 in real money balances $m$, namely in $m_{t}$ and $m_{t+1}$. Typically, at any time $t \geq 0$, we have $k_{t}$, the current capital stock, as the predetermined variable, and $m_{t}$ and $k_{t+1}$, respectively, the current real balances and the future capital stock, as the two forward variables (i.e., depending on future decisions). ${ }^{3}$

As a result, as soon as the stable manifold has dimension greater or equal to 2 , there exists a continuum of converging equilibrium paths, and the steady state is locally indeterminate. In this case, two kinds of local indeterminacy may arise:

- A local indeterminacy of order 1 when the stable manifold is twodimensional. In a given neighborhood of the steady state, the intersection of the plane defined by the forward variables $m_{t}$ and $k_{t+1}$ with the stable manifold determines a one-dimensional manifold which is the set of multiple starting points for as many equilibrium paths converging to the steady state.

\footnotetext{
${ }^{2}$ See Azariadis [1], Cass and Shell [4], Shell [10], Woodford [11].

${ }^{3}$ Indeed, while the stock of money $M_{t}$ is a predetermined variable, the real money balances $m_{t}=q_{t} M_{t}$ depend on a relative price $q_{t}$ which is a forward variable.
} 
- A local indeterminacy of order 2 when the stable manifold is threedimensional. In this case, in a given neighborhood of the steady state, the intersection of the plane defined by $m_{t}$ and $k_{t+1}$ with the stable manifold determines a two-dimensional local manifold which is the set of multiple starting points for as many converging equilibrium paths.

Most of the results available in the literature can be derived from MIUF growth models. For instance, Feenstra [5] has shown that a cash-in-advance economy can be viewed as a particular case of the MIUF approach, provided that the real balances in the utility function follow a cash-in-advance (CIA) timing. ${ }^{4}$ In this case, the preferences are assumed to depend on the cash the household has before entering the goods market, i.e. the stock of money accumulated in the previous period and available before buying new goods. In this context, there is no room for local indeterminacy of order 2 , but indeterminacy of order 1 arises provided the interest elasticity of real money demand is low enough, a condition which is hard to justify empirically.

In this paper we consider a two-sector economy with MIUF and CIA timing but we assume also some sector-specific externalities in production. We provide less stringent conditions on the preferences for the occurrence of multiple equilibria. On the one hand, we show that local indeterminacy of order 2 requires a large enough elasticity of intertemporal substitution in consumption, a low enough interest elasticity of real money demand, and a consumption good sector capital intensive at the private level but labor intensive at the social level. On the other hand, when the elasticity of intertemporal substitution in consumption is decreased, or when the interest elasticity of real money demand is increased, we prove the occurrence of local indeterminacy of order 1 through a flip bifurcation.

The paper is organized as follows: The next section sets up the basic model, defines the intertemporal equilibrium and proves the existence of a unique steady state. In section 3 , we provide our main results on local indeterminacy. Section 4 contains concluding comments and all the proofs are gathered into a final appendix.

\section{The model}

\subsection{Technology}

There are two goods: a consumption good, $c$, and a capital good, $k$. Each good is assumed to be produced with a Cobb-Douglas technology which

\footnotetext{
${ }^{4}$ See for instance Carlstrom and Fuerst [3] and Woodford [12].
} 
contains some sector specific externalities. We denote by $c$ and $y$ the outputs of sectors $c$ and $k$, and by $e_{c}$ and $e_{y}$ the corresponding external effects. The private production functions are thus defined as:

$$
c=e_{c} K_{c}^{\alpha_{1}} L_{c}^{\alpha_{2}}, y=e_{y} K_{y}^{\beta_{1}} L_{y}^{\beta_{2}}
$$

The externalities $e_{c}$ and $e_{y}$ depend on $\bar{K}_{i}$ and $\bar{L}_{i}$, which denote the average use of capital and labor in sector $i=c, y$, and are equal to

$$
e_{c}=\bar{K}_{c}^{a_{1}} \bar{L}_{c}^{a_{2}}, \quad e_{y}=\bar{K}_{y}^{b_{1}} \bar{L}_{y}^{b_{2}}
$$

with $a_{i}, b_{i} \geq 0, i=1,2$. We assume that these economy-wide averages are taken as given by individual firms. At the equilibrium, all firms of sector $i=c, y$ being identical, we have $\bar{K}_{i}=K_{i}$ and $\bar{L}_{i}=L_{i}$. Denoting $\hat{\alpha}_{i}=\alpha_{i}+a_{i}$, $\hat{\beta}_{i}=\beta_{i}+b_{i}$, the social production functions are defined as

$$
c=K_{c}^{\hat{\alpha}_{1}} L_{c}^{\hat{\alpha}_{2}}, y=K_{y}^{\hat{\beta}_{1}} L_{y}^{\hat{\beta}_{2}}
$$

We assume in the following that $\hat{\alpha}_{1}+\hat{\alpha}_{2}=1$ and $\hat{\beta}_{1}+\hat{\beta}_{2}=1$ so that the returns to scale are constant at the social level, and decreasing at the private level.

Total labor is given by $L_{c}+L_{y}=\ell$, and the total stock of capital is given by $K_{c}+K_{y}=k$. We assume complete depreciation of capital in one period so that the capital accumulation equation is $y_{t}=k_{t+1} \cdot{ }^{5}$ Optimal factor allocations across sectors are obtained by solving the following program:

$$
\begin{array}{cl}
\max _{K_{c, t}, L_{c, t}, K_{y, t}, L_{y, t}} & e_{c t} K_{c t}^{\alpha_{1}} L_{c t}^{\alpha_{2}} \\
\text { s.t. } & k_{t+1}=e_{y t} K_{y t}^{\beta_{1}} L_{y t}^{\beta_{2}} \\
& k_{t}=K_{c t}+K_{y t} \\
& e_{c t}, e_{y t} \text { given }
\end{array}
$$

Denote by $p_{t}, w_{t}$ and $r_{t}$ respectively the price of the capital good, the wage rate of labor and the rental rate of the capital good at time $t \geq 0$, all in terms of the price of the consumption good. The Lagrangian is:

$$
\begin{aligned}
L_{t} & =e_{c t} K_{c t}^{\alpha_{1}} L_{c t}^{\alpha_{2}}+w_{t}\left(\ell_{t}-L_{c t}-L_{y t}\right)+r_{t}\left(k_{t}-K_{c t}-K_{y t}\right) \\
& +p_{t}\left[e_{y t} K_{y t}^{\beta_{1}} L_{y t}^{\beta_{2}}-k_{t+1}\right]
\end{aligned}
$$

For any given $\left(k_{t}, k_{t+1}\right)$, solving the first order conditions gives input demand functions $\tilde{K}_{c}=K_{c}\left(k_{t}, k_{t+1}, e_{c t}, e_{y t}\right), \tilde{L}_{c}=L_{c}\left(k_{t}, k_{t+1}, e_{c t}, e_{y t}\right), \tilde{K}_{y}=$

${ }^{5}$ Full depreciation is introduced in order to simplify the analysis. 
$K_{y}\left(k_{t}, k_{t+1}, e_{c t}, e_{y t}\right), \tilde{L}_{y}=L_{y}\left(k_{t}, k_{t+1}, e_{c t}, e_{y t}\right)$. We then define the production frontier as

$$
c_{t}=T\left(k_{t}, k_{t+1}, e_{c t}, e_{y t}\right)=e_{c t} \tilde{K}_{c t}^{\alpha_{1}} \tilde{L}_{c t}^{\alpha_{2}}
$$

Using the envelope theorem we derive:

$$
r_{t}=T_{1}\left(k_{t}, k_{t+1}, e_{c t}, e_{y t}\right), \quad p_{t}=-T_{2}\left(k_{t}, k_{t+1}, e_{c t}, e_{y t}\right)
$$

where $T_{1}=\partial T / \partial k_{t}$ and $T_{2}=\partial T / \partial k_{t+1}$. The wage rate is then obtained as $w_{t}=T\left(k_{t}, k_{t+1}, e_{c t}, e_{y t}\right)-r_{t} k_{t}+p_{t} y_{t}$.

\section{$2.2 \quad$ Preferences}

The economy is populated by a large number of identical infinitely-lived agents. We assume without loss of generality that the total population is constant. In each period $t \geq 0$, each consumer receives income from capital and labor, and monetary lump-sum transfers from the government. His expenditures concern the consumption good, the investment good and money for the next period $t+1$. Let us then denote by $M_{t}$ the stock of money at time $t$. In each period $t \geq 0$, the representative agent is subject to the budget constraint

$$
\hat{q}_{t} c_{t}+\hat{p}_{t} k_{t+1}+M_{t+1}=\hat{r}_{t} k_{t}+\hat{w}_{t}+M_{t}+\hat{\tau}_{t}
$$

where $\hat{q}_{t}$ is the price of the consumption good, $\hat{p}_{t}$ is the price of the investment good, $\hat{r}_{t}$ is the rental rate of capital, $\hat{w}_{t}$ is the wage rate and $\hat{\tau}$ is the nominal lump-sum transfers issued by the government. Choosing the consumption good as the numéraire, i.e. dividing equation (6) by $\hat{q}_{t}$, gives a reformulation of the budget constraint

$$
c_{t}+p_{t} k_{t+1}+q_{t} M_{t+1}=r_{t} k_{t}+w_{t}+q_{t} M_{t}+\tau_{t}
$$

with $p_{t}=\hat{p}_{t} / \hat{q}_{t}, q_{t}=1 / \hat{q}_{t}, r_{t}=\hat{r}_{t} / \hat{q}_{t}, w_{t}=\hat{w}_{t} / \hat{q}_{t}$ and $\tau_{t}=\hat{\tau}_{t} / \hat{q}_{t}$. Money in real terms is thus given by $q_{t} M_{t}$.

The per-period utility function of the representative agents depends on consumption $c$ and real money balances $q \mathcal{M}$. With the CIA timing, the amount of money that facilitates trading at time $t$ corresponds to the cash the household has in advance of goods-market trading, namely

$$
\mathcal{M}_{t}=M_{t}
$$

Let us then denote real money balances at time $t$ by $m_{t}=q_{t} M_{t}$.

We assume that the utility function is additively-separable between consumption and real money balances:

$$
U(c, m)=u(c)+v(m)
$$


Assumption 1. $u(c)$ and $v(m)$ are $C^{2}$, increasing, concave and satisfies the boundary conditions $\lim _{c \rightarrow 0} u^{\prime}(c)=+\infty, \lim _{m \rightarrow 0} v^{\prime}(m)=+\infty$, $\lim _{c \rightarrow+\infty} u^{\prime}(c)=0, \lim _{m \rightarrow+\infty} v^{\prime}(m)=0$.

Notice that the production frontier $T\left(k, y, e_{c}, e_{y}\right)$ gives the maximum production level of the consumption good which will be entirely consumed by the representative agent, i.e. $c_{t}=T\left(k_{t}, k_{t+1}, e_{c t}, e_{y t}\right)$. The intertemporal maximisation program of the representative agent is then as follows

$$
\begin{array}{cl}
\max _{\left\{c_{t}, k_{t+1}, M_{t+1}\right\}_{t=0}^{+\infty}} & \sum_{t=0}^{+\infty} \delta^{t}\left[u\left(c_{t}\right)+v\left(q_{t} M_{t}\right)\right] \\
\text { s.t. } & c_{t}+p_{t} k_{t+1}+q_{t} M_{t+1}=r_{t} k_{t}+w_{t}+q_{t} M_{t}+\tau_{t} \\
& k_{0}, M_{0} \text { given }
\end{array}
$$

where $\delta \in(0,1]$ denotes the discount factor.

\subsection{Intertemporal equilibrium}

The generalised Lagrangian corresponding to the intertemporal optimization problem (9) is

$$
\begin{aligned}
\mathcal{L} & =\sum_{t=0}^{+\infty} \delta^{t}\left[u\left(c_{t}\right)+v\left(q_{t} M_{t}\right)\right] \\
& +\sum_{t=0}^{+\infty} \delta^{t} \lambda_{t}\left[r_{t} k_{t}+w_{t}+q_{t} M_{t}+\tau_{t}-c_{t}-p_{t} k_{t+1}-q_{t} M_{t+1}\right]
\end{aligned}
$$

with $\lambda$ the non-negative Lagrange multiplier associated with constraint (7). The first-order conditions with respect to $c_{t}, k_{t+1}$ and $M_{t+1}$ are given by

$$
\begin{aligned}
\lambda_{t} & =u^{\prime}\left(c_{t}\right) \\
\lambda_{t} p_{t} & =\delta \lambda_{t+1} r_{t+1} \\
\lambda_{t} q_{t} & =\delta q_{t+1}\left[\lambda_{t+1}+v^{\prime}\left(q_{t+1} M_{t+1}\right)\right]
\end{aligned}
$$

with $r_{t}$ and $p_{t}$ as defined by (5). By manipulating (11)-(13), we get the standard Euler equation

$$
u^{\prime}\left(c_{t}\right) p_{t}=\delta u^{\prime}\left(c_{t+1}\right) r_{t+1}
$$

and the arbitrage condition between consumption and real balances

$$
u^{\prime}\left(c_{t}\right) q_{t}=\delta q_{t+1}\left[u^{\prime}\left(c_{t+1}\right)+v^{\prime}\left(q_{t+1} M_{t+1}\right)\right]
$$

From the input demand functions defined in Section 2.1 together with the external effects (2) considered at the equilibrium, we define the equilibrium factors demand functions $\hat{K}_{c}=\hat{K}_{c}\left(k_{t}, k_{t+1}\right), \hat{K}_{y}=\hat{K}_{y}\left(k_{t}, k_{t+1}\right)$, 
$\hat{L}_{c}=\hat{L}_{c}\left(k_{t}, k_{t+1}\right), \hat{L}_{y}=\hat{L}_{y}\left(k_{t}, k_{t+1}\right)$ so that $\hat{e}_{c}=\hat{e}_{c}\left(k_{t}, k_{t+1}\right)=\hat{K}_{c}^{a_{1}} \hat{L}_{c}^{a_{2}}$ and $\hat{e}_{y}=\hat{e}_{y}\left(k_{t}, k_{t+1}\right)=\hat{K}_{y}^{b_{1}} \hat{L}_{y}^{b_{2}}$. From (5), prices now satisfy

$$
\begin{aligned}
& r\left(k_{t}, k_{t+1}\right)=T_{1}\left(k_{t}, k_{t+1}, \hat{e}_{c}\left(k_{t}, k_{t+1}\right), \hat{e}_{y}\left(k_{t}, k_{t+1}\right)\right) \\
& p\left(k_{t}, k_{t+1}\right)=-T_{2}\left(k_{t}, k_{t+1}, \hat{e}_{c}\left(k_{t}, k_{t+1}\right), \hat{e}_{y}\left(k_{t}, k_{t+1}\right)\right)
\end{aligned}
$$

and the consumption level at time $t$ is given by

$$
c\left(k_{t}, k_{t+1}\right)=T\left(k_{t}, k_{t+1}, \hat{e}_{c}\left(k_{t}, k_{t+1}\right), \hat{e}_{y}\left(k_{t}, k_{t+1}\right)\right)
$$

In order to complete the definition of the intertemporal equilibrium, we need to specify the supply of money. Government follows a simple monetary rule: in each period it issues lump-sum transfers of money balances at the constant rate $\theta-1>0$, so that in period $t$ the supply of money $M_{t}^{s}$ satisfies $M_{t}^{s}=\theta^{t} M_{0}^{s}$, with $M_{0}^{s}=M_{0}$ the initial amount of money balances. Thus nominal transfers are given by $\tau_{t}=(\theta-1) q_{t} M_{t}^{s}$. We then get the first order conditions (14), (15) evaluated at $\hat{e}_{c}$ and $\hat{e}_{y}$, namely the Euler equation

$$
u^{\prime}\left(c\left(k_{t}, k_{t+1}\right)\right) p\left(k_{t}, k_{t+1}\right)=\delta u^{\prime}\left(c\left(k_{t+1}, k_{t+2}\right)\right) r\left(k_{t+1}, k_{t+2},\right)
$$

and the arbitrage condition between consumption and real balances

$$
u^{\prime}\left(c\left(k_{t}, k_{t+1}\right)\right) m_{t}=\frac{\delta}{\theta} m_{t+1}\left[u^{\prime}\left(c\left(k_{t+1}, k_{t+2}\right)\right)+v^{\prime}\left(m_{t+1}\right)\right]
$$

Any solution $\left\{\left(k_{t}, m_{t}\right)\right\}_{t=0}^{+\infty}$ which also satisfies the transversality conditions

$$
\begin{aligned}
\lim _{t \rightarrow+\infty} \delta^{t} u^{\prime}\left(c\left(k_{t}, k_{t+1}\right)\right) p\left(k_{t}, k_{t+1}\right) k_{t+1} & =0 \\
\lim _{t \rightarrow+\infty} \delta^{t} u^{\prime}\left(c\left(k_{t}, k_{t+1}\right)\right) m_{t} & =0
\end{aligned}
$$

is called an equilibrium path.

\subsection{Steady state}

A steady state is defined as $k_{t}=k^{*}$ and $m_{t}=m^{*}$ for all $t$. Along a stationary equilibrium, we have indeed a decreasing path for the price of money balances $q_{t+1} / q_{t}=1 / \theta$ which is associated with the expansion of money supply $M_{t+1} / M_{t}=\theta$ so that real money balances $m_{t}$ remain constant. Notice also that the steady state value of consumption $c^{*}$ is directly obtained from $k^{*}$ through the production frontier, namely $c^{*}=$ $T\left(k^{*}, k^{*}, \hat{e}_{c}\left(k^{*}, k^{*}, \hat{e}_{y}\left(k^{*}, k^{*}\right)\right) \equiv T^{*}\right.$. The stationary levels of capital $k^{*}$ and real money balances $m^{*}$ are obtained as solutions of

$$
\frac{r(k, k)}{p(k, k)}=\delta^{-1} \text { and } \frac{v^{\prime}(m)}{u^{\prime}(c)}=\frac{\theta-\delta}{\delta}>0
$$


Notice that the stationary capital stock $k^{*}$ only depends on the characteristics of the technologies and is independent from the per-period utility function. As a result, once the existence of $k^{*}$ is ensured, we get $c^{*}=T^{*}$ and the stationary real money balances $m^{*}$ are directly obtained from the per-period utility function associated with money holding. Then we get the following conclusion:

Proposition 1. Under Assumption 1, there exists a unique steady state $\left(k^{*}, m^{*}\right)$.

Proof: See Appendix 5.1

In the following, $\left(k^{*}, m^{*}\right)$ will be called the Modified Golden Rule.

\section{Main results}

In order to provide a clear-cut local stability analysis of the equilibrium paths in a neighborhood of the Modified Golden Rule, we specify the per-period utility function with respect to consumption as follows:

$$
u(c)=\frac{c^{1-\sigma}}{1-\sigma}
$$

with $\sigma \geq 0$. Hence, the elasticity of intertemporal substitution in consumption is given by $\epsilon_{c}=1 / \sigma$.

With such a formulation, we derive the characteristic polynomial building on some results provided in Nishimura and Venditti [8]. Let us introduce the interest elasticity of real money demand evaluated at the steady state:

$$
\varepsilon_{m}=-\frac{v^{\prime}\left(m^{*}\right)}{v^{\prime \prime}\left(m^{*}\right) m^{*}}>0
$$

Linearizing equations (18) and (19) around $\left(k^{*}, m^{*}\right)$ gives:

Proposition 2. The characteristic polynomial is

$$
\begin{aligned}
\mathcal{P}(x) & =\left(x-\frac{\theta \varepsilon_{m}}{\theta \varepsilon_{m}-(\theta-\beta)}\right)\left[\left(x-\frac{\alpha_{2}}{\delta\left(\alpha_{2} \beta_{1}-\alpha_{1} \beta_{2}\right)}\right)\left(x-\frac{\hat{\beta}_{1}-\hat{\alpha}_{1}}{\hat{\alpha}_{2}}\right)\right. \\
& \left.-\sigma\left(\frac{\left(1-\delta \beta_{1}\right)\left(\hat{\alpha}_{2} \alpha_{1} \beta_{2}+\hat{\alpha}_{1} \alpha_{2} \beta_{1}\right)+\delta \beta_{1} \alpha_{1} \beta_{2}}{\hat{\alpha}_{2}\left(1-\delta \beta_{1}\right)\left(\alpha_{2} \beta_{1}-\alpha_{1} \beta_{2}\right)} x-\frac{\left(1-\delta \beta_{1}\right) \hat{\alpha}_{1} \alpha_{2}+\delta \hat{\beta}_{1} \alpha_{1} \beta_{2}}{\delta \hat{\alpha}_{2}\left(1-\delta \beta_{1}\right)\left(\alpha_{2} \beta_{1}-\alpha_{1} \beta_{2}\right)}\right)(x-1)\right] \\
& \equiv(x-\Lambda) \tilde{\mathcal{P}}(x)
\end{aligned}
$$

Proof: See Appendix 5.2.

Proposition 2 shows that the first characteristic root $x_{1}=\Lambda$ depends on the properties of the utility derived from money holding, while the two others, $x_{2}$ and $x_{3}$, depend on the parameters of the technologies and $\sigma$.

Let us start with the first characteristic root $x_{1}$. The following Lemma provides a precise characterization: 
Lemma 1. Under Assumption 1, $x_{1} \in(-1,0)$ if and only if $\varepsilon_{m} \in(0,(\theta-$ $\beta) / 2 \theta)$.

Proof: Obvious from Proposition 2.

Let us consider now the two other roots $x_{2}$ and $x_{3}$. Note that if the utility function is linear in consumption, i.e. $\sigma=0$, these two roots are

$$
x_{2}=\frac{\alpha_{2}}{\delta\left(\alpha_{2} \beta_{1}-\alpha_{1} \beta_{2}\right)}, \quad x_{3}=\frac{\hat{\beta}_{1}-\hat{\alpha}_{1}}{\hat{\alpha}_{2}}<1
$$

We then have

$$
\begin{aligned}
& x_{2} \in(-1,0) \quad \Leftrightarrow \quad \alpha_{1} \beta_{2}-\alpha_{2} \beta_{1}>\alpha_{2} / \delta \\
& x_{3} \in(-1,1) \quad \Leftrightarrow \quad \hat{\beta}_{1}>\hat{\alpha}_{1}-\hat{\alpha}_{2}
\end{aligned}
$$

These conditions have clear economic interpretations. Based on the private and social production functions as defined respectively by (1) and (3), factor intensities are determined by the parameters of the Cobb-Douglas technologies. If $\alpha_{1} \beta_{2}>(<) \alpha_{2} \beta_{1}$, the consumption (investment) good sector is capital intensive at the private level, and if $\hat{\alpha}_{1}>\hat{\beta}_{1}$ the consumption (investment) good sector is capital intensive at the social level. Then, the first condition requires that the consumption good is suffiently capital intensive at the private level. The second condition holds if the consumption good is labor intensive at the social level but can be also satisfied even if the consumption good is also capital intensive at the social level.

When the utility function is non-linear, i.e. if $\sigma>0$, it can be shown that under the conditions given in $(24)$, we still get $x_{2} \in(-1,0)$ and $x_{3} \in(-1,1)$ provided the value of $\sigma$ is not too large. More precisely, we have:

Lemma 2. Under Assumption 1, let $\alpha_{1} \beta_{2}-\alpha_{2} \beta_{1}>\alpha_{2} / \delta$ and $\hat{\beta}_{1}>\hat{\alpha}_{1}-\hat{\alpha}_{2}$. Then $x_{2} \in(-1,0)$ and $x_{3} \in(-1,1)$ if and only if $\sigma \in\left[0, \sigma^{*}\right)$ with

$$
\sigma^{*}=\frac{\left[\delta\left(\alpha_{1} \beta_{2}-\alpha_{2} \beta_{1}\right)-\alpha_{2}\right]\left[\hat{\beta}_{1}-\left(\hat{\alpha}_{1}-\hat{\alpha}_{2}\right)\right]\left(1-\delta \beta_{1}\right)}{2\left[\left(1-\delta \beta_{1}\right)\left[\hat{\alpha}_{1} \alpha_{2}\left(1+\delta \beta_{1}\right)+\delta \hat{\alpha}_{2} \alpha_{1} \beta_{2}\right]+\delta \alpha_{1} \beta_{2}\left(\hat{\beta}_{1}+\delta \beta_{1}\right)\right]}
$$

Proof: See Appendix 5.3.

Building on these results, we may now discuss the multiplicity of equilibrium paths. As seen in the Introduction, local indeterminacy arises when the stable manifold has dimension greater than the number of predetermined variables. Equations (18) and (19) define a system of implicit difference equations of order 2 in the capital stock $k$ and order 1 in the real money balances $m$. Since $k_{t}$ has been decided at period $t-1$, it plays the role of predetermined variable at time $t$ while, on the contrary, $m_{t}$ and $k_{t+1}$ can be chosen at time $t$ and play the role of forward variables. More precisely, 
even if the stock of money $M_{t}$ is a predetermined variable, the real balances $m_{t}=q_{t} M_{t}$ inherit the status of forward variable from the relative price $q_{t}$.

As a result, the equilibrium is unique if one characteristic root is stable, i.e. belongs to the unit circle, and two characteristic roots are unstable, i.e. lie outside the unit circle. In other words, uniqueness rests on the existence of one-dimensional stable manifold. Conversely, as soon as the stable manifold has dimension greater or equal to 2 , there exists a continuum of converging equilibrium paths and the steady state is locally indeterminate. In this case, two kinds of local indeterminacy may arise:

- A local indeterminacy of order 1 when the stable manifold is twodimensional. In a given neighborhood of the steady state, the intersection of the plane defined by the forward variables $m_{t}$ and $k_{t+1}$ with the stable manifold is a one-dimensional manifold. From each point of this manifold starts an equilibrium trajectory converging to the steady state.

- A local indeterminacy of order 2 when the stable manifold is threedimensional. Indeed, in a given neighborhood of the steady state, the intersection of the plane defined by $m_{t}$ and $k_{t+1}$ with the stable manifold gives a two-dimensional local manifold which contains multiple starting points for as many converging equilibrium paths.

The following Proposition provides clear-cut conditions for the existence of local indeterminacy of both the orders.

Proposition 3. Let Assumption 1 holds and consider the critical bound $\sigma^{*}$ as defined by (25). Then the steady state is locally indeterminate of order 2 if and only if $\alpha_{1} \beta_{2}-\alpha_{2} \beta_{1}>\alpha_{2} / \delta, \hat{\beta}_{1}>\hat{\alpha}_{1}-\hat{\alpha}_{2}, \sigma \in\left[0, \sigma^{*}\right)$ and $\varepsilon_{m} \in(0,(\theta-\beta) / 2 \theta)$. Moreover:

i) For any given $\varepsilon_{m} \in(0,(\theta-\beta) / 2 \theta)$, the steady state is locally indeterminate of order 1 when $\sigma>\sigma^{*}$, where $\sigma^{*}$ is a flip bifurcation value giving rise to period-two cycles. If the cycles of period 2 arise in the right (respectively, left) neighbourhood of $\sigma^{*}$, they are locally indeterminate of order 2 (respectively, locally indeterminate of order 1 ).

ii) For any given $\sigma \in\left[0, \sigma^{*}\right)$, the steady state is locally indeterminate of order 1 when $\varepsilon_{m}>\varepsilon_{m}^{*}=(\theta-\beta) / 2 \theta$, where $\varepsilon_{m}^{*}$ is a flip bifurcation value giving rise to period-two cycles. If the cycles of period 2 arise in the right (respectively, left) neighbourhood of $\varepsilon_{m}^{*}$, they are locally indeterminate of order 2 (respectively, locally indeterminate of order 1 ).

Proof: See Appendix 5.4.

Proposition 3 shows that under some appropriate restrictions on the capital intensity differences at the private and social levels, a large enough elastic- 
ity of intertemporal substitution in consumption and a low enough interest elasticity of real money demand are necessary and sufficient conditions for the occurrence of local indeterminacy on the full dimensional phase space. Moreover, when the elasticity of intertemporal substitution in consumption is decreased, or when the interest elasticity of real money demand is increased, we prove the occurrence of local indeterminacy of order 1 through a flip bifurcation. Case ii) is particularly worthwhile as it is based on a large enough interest elasticity of real money demand, a condition that matches empirical evidences. We also show that period-two cycles are intimately connected with the existence of multiple equilibria.

\section{Concluding comments}

We have shown that local indeterminacy and business-cycle fluctuations based on self-fulfilling prophecies occur in a two-sector monetary economy with productive externalities and money in an additively separable utility function. More precisely, we have provided conditions on technologies leading to the existence of local indeterminacy for any value of the interest rate elasticity of money demand, provided the elasticity of intertemporal substitution in consumption is large enough. Indeed, while local indeterminacy of order 2 requires a low enough interest elasticity of real money demand, local indeterminacy of order 1 can be obtained even with a large interest elasticity of real money demand. However, as in this last case the restriction on the elasticity of intertemporal substitution in consumption appears to be disputed with respect to empirical evidences, it would be interesting to generalize the formulation of preferences by considering a non-separable utility function and to check whether this condition could be relaxed. This is left for future research.

\section{Appendix}

\subsection{Proof of Proposition 1}

Consider first equation (22). We need to compute the partial derivatives of $T$. From the Lagrangian (10) we derive the first order conditions:

$$
\begin{aligned}
r & =e_{c} \alpha_{1} K_{c}^{\alpha_{1}-1} L_{c}^{\alpha_{2}}=p e_{y} \beta_{1} K_{y}^{\beta_{1}-1} L_{y}^{\beta_{2}} \\
w & =e_{c} \alpha_{2} K_{c}^{\alpha_{1}} L_{c}^{\alpha_{2}-1}=p e_{y} \beta_{2} K_{y}^{\beta_{1}} L_{y}^{\beta_{2}-1}
\end{aligned}
$$

Using $K_{c}=k_{0}-K_{y}, L_{y}=1-L_{c}$, and merging the above equations gives: 


$$
\begin{aligned}
L_{c}^{*} & =\frac{\alpha_{2} \beta_{1}\left(k_{0}-K_{y}^{*}\right)}{\left(\alpha_{1} \beta_{2}-\alpha_{2} \beta_{1}\right) K_{y}^{*}+\alpha_{2} \beta_{1} k_{0}} \\
L_{y}^{*} & =\frac{\alpha_{1} \beta_{2} K_{y}^{*}}{\left(\alpha_{1} \beta_{2}-\alpha_{2} \beta_{1}\right) K_{y}^{*}+\alpha_{2} \beta_{1} k_{0}} \\
K_{c}^{*} & =k_{0}-K_{y}^{*} \\
K_{y}^{*} & =g\left(k_{0}, k_{1}\right) \equiv g
\end{aligned}
$$

where

$$
g\left(k_{0}, k_{1}\right)=\left\{K_{y} \in\left[0, e_{y} k^{\beta_{1}}\right] / k_{1}=\frac{e_{y}\left(\alpha_{1} \beta_{2}\right)^{\beta_{2}} K_{y}^{\beta_{1}+\beta_{2}}}{\left[\alpha_{2} \beta_{1} k_{0}+\left(\alpha_{1} \beta_{2}-\alpha_{2} \beta_{1}\right) K_{y}\right]^{\beta_{2}}}\right\}
$$

To simplify notation let:

$$
\Delta=\left(\alpha_{1} \beta_{2}-\alpha_{2} \beta_{1}\right) g+\alpha_{2} \beta_{1} k_{0}
$$

From the envelope theorem we get:

$$
T_{1}=r^{*}, \quad T_{2}=-p^{*}
$$

From (26), (28) and (30) we obtain:

$$
r^{*}=e_{c} \alpha_{1}\left(k_{0}-g\right)^{\alpha_{1}+\alpha_{2}-1}\left(\alpha_{2} \beta_{1}\right)^{\alpha_{2}} \Delta^{-\alpha_{2}}
$$

and from (26), (29), (31) and (34):

$$
p^{*}=\frac{e_{c} \alpha_{1}}{e_{y} \beta_{1}}\left(\alpha_{2} \beta_{1}\right)^{\alpha_{2}}\left(\alpha_{1} \beta_{2}\right)^{-\beta_{2}}\left(k_{0}-g\right)^{\alpha_{1}+\alpha_{2}-1} g^{1-\beta_{1}-\beta_{2}} \Delta^{\beta_{2}-\alpha_{2}}
$$

By the derivation of $g$, we have, for any equilibrium path, the identity $\Delta=e_{y}^{1 / \beta_{2}} \alpha_{1} \beta_{2}(g / y)^{1 / \beta_{2}}$. Substituting this into (34) and (35) gives after simplifications:

$T_{1}\left(k_{0}, k_{1}, e_{c}, e_{y}\right)=\alpha_{1} e_{c} e_{y}^{-\frac{\alpha_{2}}{\beta_{2}}}\left(\frac{\alpha_{2} \beta_{1}}{\alpha_{1} \beta_{2}}\right)^{\alpha_{2}}\left(k_{0}-g\right)^{\alpha_{1}+\alpha_{2}-1} g^{-\frac{\alpha_{2}\left(\beta_{1}+\beta_{2}\right)}{\beta_{2}}} y^{\frac{\alpha_{2}}{\beta_{2}}}$

$T_{2}\left(k_{0}, k_{1}, e_{c}, e_{y}\right)=-\frac{\alpha_{1}}{\beta_{1}} e_{c} e_{y}^{-\frac{\alpha_{2}}{\beta_{2}}}\left(\frac{\alpha_{2} \beta_{1}}{\alpha_{1} \beta_{2}}\right)^{\alpha_{2}}\left(k_{0}-g\right)^{\alpha 1+\alpha 2-1} g^{\frac{\beta_{2}-\alpha_{2}\left(\beta_{1}+\beta_{2}\right)}{\beta_{2}}} y^{\frac{\alpha_{2}-\beta_{2}}{\beta_{2}}}$

A steady state $k^{*}$ is then defined as $T_{1}\left(k^{*}, k^{*}, e_{c}^{*}, e_{y}^{*}\right)+\rho T_{2}\left(k^{*}, k^{*}, e_{c}^{*}, e_{y}^{*}\right)$ with $e_{c}^{*}=\hat{e}_{c}\left(k^{*}, k^{*}\right)$ and $e_{y}^{*}=\hat{e}_{y}\left(k^{*}, k^{*}\right)$. Denote $g^{*}=g\left(k^{*}, k^{*}\right)$ and $y^{*}=k^{*}$. Using the derivatives of $T$ in the definition of $k^{*}$ gives:

$$
g^{*}=\delta \beta_{1} k^{*}
$$

Substituting (36) into the definition of $g$, we find

$$
k^{*}=e_{y}^{*}\left(\alpha_{1} \beta_{2}\right)^{\beta_{2}}\left(\delta \beta_{1} k^{*}\right)^{\beta_{1}+\beta_{2}}\left[\left(\alpha_{1} \beta_{2}-\alpha_{2} \beta_{1}\right) \delta \beta_{1} k^{*}+\alpha_{2} \beta_{1} k^{*}\right]^{-\beta_{2}}
$$


Considering that:

$$
e_{y}^{*}=\left(\delta \beta_{1} k^{*}\right)^{b_{1}+b_{2}}\left(\alpha_{1} \beta_{2}\right)^{b_{2}}\left[\left(\alpha_{1} \beta_{2}-\alpha_{2} \beta_{1}\right) \delta \beta_{1} k^{*}+\alpha_{2} \beta_{1} k^{*}\right]^{-b_{2}}
$$

and substituting $e_{y}^{*}$ into the above equation, finally gives

$$
k^{*}=\frac{\alpha_{1} \beta_{2}}{\beta_{1}\left[\alpha_{2}+\delta\left(\alpha_{1} \beta_{2}-\alpha_{2} \beta_{1}\right)\right]}\left(\delta \beta_{1}\right)^{\frac{1}{\hat{\beta}_{2}}}
$$

Consider now the monetary part of equation (22) evaluated at $k^{*}$ with $c^{*}=T^{*}$. We get:

$$
v^{\prime}(m)=\frac{\theta-\delta}{\delta} u^{\prime}\left(T^{*}\right)
$$

The boundary conditions in Assumption 1 guarantee the existence and uniqueness of a solution $m^{*}$.

\subsection{Proof of Proposition 2}

Total differentiation of equations (18) and (19) gives after tedious computations available upon request

$$
\begin{aligned}
& d k_{t+2}\left[1+\sigma \frac{T_{1}^{* 2}}{c^{*} \mathcal{T}_{12}^{*}} \delta \mathcal{B}\right]+d k_{t+1}\left[\frac{\mathcal{T}_{22}^{*}}{\delta \mathcal{T}_{12}^{*}}+\frac{\mathcal{T}_{11}^{*}}{\mathcal{T}_{12}^{*}}-\sigma \frac{T_{1}^{* 2}}{c^{*} \mathcal{T}_{12}^{*}}(\delta \mathcal{B}+\mathcal{A})\right]+d k_{t}\left[\frac{\mathcal{T}_{22}^{*}}{\delta \mathcal{T}_{12}^{*}} \frac{\mathcal{T}_{11}^{*}}{\mathcal{T}_{12}^{*}}+\sigma \frac{T_{1}^{* 2}}{c^{*} \mathcal{T}_{12}^{*}} \mathcal{A}\right]=0 \\
& d k_{t+2} T_{1} \delta \mathcal{B}-d k_{t+1} T_{1}(\mathcal{A}+\delta \mathcal{B})+d k_{t} \frac{\theta}{\delta} T_{1} \mathcal{A}+d m_{t+1} \frac{c^{*}\left(\theta \varepsilon_{m}-(\theta-\beta)\right)}{\delta \sigma m^{*} \varepsilon_{m}}-d m_{t} \frac{\theta c^{*}}{\delta \sigma m^{*}}=0
\end{aligned}
$$

with

$$
\begin{aligned}
& \frac{\mathcal{T}_{11}^{*}}{\mathcal{T}_{12}^{*}}=-\frac{\alpha_{2}}{\delta\left(\alpha_{2} \beta_{1}-\alpha_{1} \beta_{2}\right)}, \frac{\mathcal{T}_{22}^{*}}{\delta \mathcal{T}_{12}^{*}}=-\frac{\hat{\beta}_{1}-\hat{\alpha}_{1}}{\hat{\alpha}_{2}}, \frac{T_{1}^{* 2}}{c^{*} \mathcal{T}_{12}^{*}}=-\frac{\alpha_{1}}{\hat{\alpha}_{2}} \frac{\mathcal{T}_{11}^{*}}{\mathcal{T}_{12}^{*}} \frac{1+\hat{\beta}_{1} \frac{\mathcal{T}_{12}^{*}}{\mathcal{T}_{11}^{*}}}{1-\delta \beta_{1}} \\
& \mathcal{A}=\frac{\hat{\alpha}_{1}}{\alpha_{1}} \frac{1-\delta \beta_{1}+\delta \beta_{1} \frac{\hat{\alpha}_{2} \hat{\beta}_{1}}{\hat{\alpha}_{1} \hat{\beta}_{2}} \frac{\alpha_{1} \beta_{2}}{\alpha_{2} \beta_{1}}\left(1+\frac{\mathcal{T}_{22}^{*}}{\delta \mathcal{T}_{12}^{*}}\right)}{1+\hat{\beta}_{1} \frac{\mathcal{T}_{12}^{*}}{\mathcal{T}_{11}^{*}}}, \mathcal{B}=\frac{\hat{\alpha}_{2}}{\alpha_{2}} \frac{\beta_{2}}{\hat{\beta}_{2}} \frac{\left(1-\delta \beta_{1}\right)\left[1-\hat{\beta}_{1}\left(1-\frac{\hat{\alpha}_{1} \hat{\beta}_{2}}{\hat{\alpha}_{2} \hat{\beta}_{1}} \frac{\alpha_{2} \beta_{1}}{\alpha_{1} \beta_{2}}\right)\right]+\delta \beta_{1}\left(1+\frac{\mathcal{T}_{22}^{*}}{\delta T_{12}^{*}}\right)}{1+\hat{\beta}_{12}} \frac{\mathcal{T}_{12}^{*}}{\mathcal{T}_{11}^{*}}
\end{aligned}
$$

Then we derive the associated Jacobian matrix:

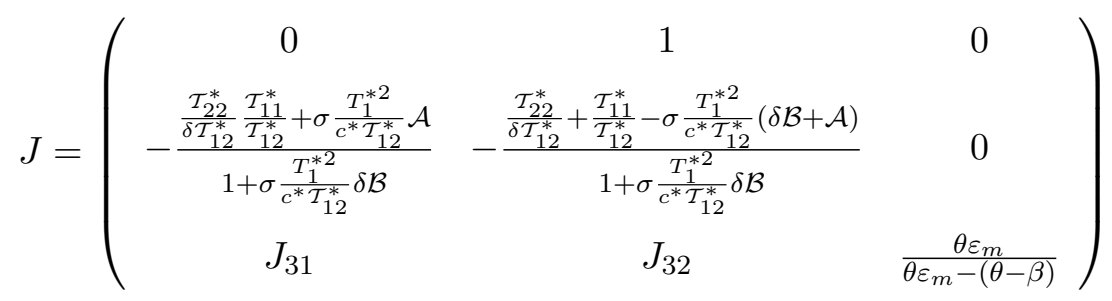

The result follows after straightforward simplifications. 


\subsection{Proof of Lemma 2}

Consider the expression between brackets in the characteristic polynomial given in Proposition 2, namely:

$$
\begin{aligned}
\tilde{\mathcal{P}}(x) & =\left[\left(x-\frac{\alpha_{2}}{\delta\left(\alpha_{2} \beta_{1}-\alpha_{1} \beta_{2}\right)}\right)\left(x-\frac{\hat{\beta}_{1}-\hat{\alpha}_{1}}{\hat{\alpha}_{2}}\right)\right. \\
& \left.-\sigma\left(\frac{\left(1-\delta \beta_{1}\right)\left(\hat{\alpha}_{2} \alpha_{1} \beta_{2}+\hat{\alpha}_{1} \alpha_{2} \beta_{1}\right)+\delta \beta_{1} \alpha_{1} \beta_{2}}{\hat{\alpha}_{2}\left(1-\delta \beta_{1}\right)\left(\alpha_{2} \beta_{1}-\alpha_{1} \beta_{2}\right)} x-\frac{\left(1-\delta \beta_{1}\right) \hat{\alpha}_{1} \alpha_{2}+\delta \hat{\beta}_{1} \alpha_{1} \beta_{2}}{\delta \hat{\alpha}_{2}\left(1-\delta \beta_{1}\right)\left(\alpha_{2} \beta_{1}-\alpha_{1} \beta_{2}\right)}\right)(x-1)\right]
\end{aligned}
$$

Under the conditions given in $(24)$, we get $x_{2} \in(-1,0)$ and $x_{3} \in(-1,1)$ when $\sigma=0$. When $\sigma$ increases the two roots decrease and the loss of stability is obtained when one root goes outside the unit circle through -1 . The bifurcation value of $\sigma$, denoted $\sigma^{*}$, is then obtained by solving $\tilde{\mathcal{P}}(-1)=0$.

\subsection{Proof of Proposition 3}

The first part is immediately derived from Lemmas 1 and 2 . The existence of a flip bifurcation is generically obtained when one characteristic root crosses the value -1 , which happens, for any given $\varepsilon_{m} \in(0,(\theta-\beta) / 2 \theta)$, when $\sigma$ crosses $\sigma^{*}$ from below. In addition, period-two cycles appear either in a right or left neighbourhood of $\sigma^{*}$ according to the properties of the nonlinear part of the system of difference equations, while their stability properties depend on the stability properties of the steady state (see Ruelle [9]). Therefore, it follows that, generically, $\sigma^{*}$ is a flip bifurcation value giving rise to periodtwo cycles for any $\sigma$ in its right (resp. left) neighborhood, which are locally indeterminate of order 2 (resp. locally indeterminate of order 1 ). This proves case $i$ ). Case $i i$ ) is proved with a similar argument for any given $\sigma \in\left[0, \sigma^{*}\right)$ using $\varepsilon_{m}$ as bifurcation parameter. In this case, $\varepsilon_{m}^{*}=(\theta-\beta) / 2 \theta$ is also a flip bifurcation value.

\section{References}

[1] Azariadis, C. (1981): "Self Fulfilling Prophecies", Journal of Economic Theory, 25, 380-396.

[2] Benhabib, J., and K. Nishimura (1998): "Indeterminacy and Sunspots with Constant Returns," Journal of Economic Theory, 81, 58-96. 
[3] Carlstrom, C., and T. Fuerst (2001): "Timing and Real Indeterminacy in Monetary Models," Journal of Monetary Economics, 47, 285-298.

[4] Cass, D., and K. Shell (1983): "Do Sunspots Matter ?," Journal of Political Economy, 91, 193-227.

[5] Feenstra, R. (1986): "Functional Equivalence Between Liquidity Costs and the Utility of Money," Journal of Monetary Economics, 17, 271291.

[6] Fukuda, S. (1993): "The Emergence of Equilibrium Cycles in a Monetary Economy with a Separable Utility Function," Journal of Monetary Economy, 32, 321-334.

[7] Matsuyama, K. (1990): "Sunspot Equilibria (Rational Bubbles) in a Model of Money-in-the-Utility-Function," Journal of Monetary Economics, 25, 137-144.

[8] Nishimura, K., and A. Venditti (2007): "Indeterminacy in DiscreteTime Infinite-Horizon Models with Non-Linear Utility and Endogenous Labor," Journal of Mathematical Economics, 43, 446-476.

[9] Ruelle, D. (1989): Elements of Differentiable Dynamics and Bifurcation Theory, Academic Press, San Diego.

[10] Shell, K. (1977): "Monnaie et Allocation Intertemporelle", mimeo, Séminaire d'Econométrie Roy-Malinvaud, Centre National de la Recherche Scientifique, Paris.

[11] Woodford, M. (1986): "Stationary Sunspot Equilibria: the Case of Small Fluctuations Around a Deterministic Steady State", Mimeo, University of Chicago.

[12] Woodford, M. (1994): "Monetary Policy and Price Level Determinacy in a Cash-in-Advance Economy," Economic Theory, 4, 345-380. 